\title{
ACCURACY ASSESSMENT OF MODELING ARCHITECTURAL STRUCTURES AND DETAILS USING TERRESTRIAL LASER SCANNING
}

\author{
M. Kedzierski ${ }^{\text {a }}$, P.Walczykowski ${ }^{\text {a }}$, A.Orych a *, P. Czarnecka ${ }^{\text {a }}$ \\ ${ }^{a}$ Department of Remote Sensing and Photogrammetry, Geodesy Institute, Faculty of Civil Engineering and Geodesy, Military \\ University of Technology, Warsaw, Poland - (mkedzierski, pwalczykowski, aorych)@wat.edu.pl, paulina.czarnecka.itfn@gmail.com
}

Commission III, WG III/2

KEY WORDS: Laser Scanning, 3D model, Accuracy, Historical Structures, Materials, Test

\begin{abstract}
:
One of the most important aspects when performing architectural documentation of cultural heritage structures is the accuracy of both the data and the products which are generated from these data: documentation in the form of 3D models or vector drawings. The paper describes an assessment of the accuracy of modelling data acquired using a terrestrial phase scanner in relation to the density of a point cloud representing the surface of different types of construction materials typical for cultural heritage structures. This analysis includes the impact of the scanning geometry: the incidence angle of the laser beam and the scanning distance. For the purposes of this research, a test field consisting of samples of different types of construction materials (brick, wood, plastic, plaster, a ceramic tile, sheet metal) was built. The study involved conducting measurements at different angles and from a range of distances for chosen scanning densities. Data, acquired in the form of point clouds, were then filt(ered and modelled. An accuracy assessment of the 3D model was conducted by fitting it with the point cloud. The reflection intensity of each type of material was also analyzed, trying to determine which construction materials have the highest reflectance coefficients, and which have the lowest reflection coefficients, and in turn how this variable changes for different scanning parameters. Additionally measurements were taken of a fragment of a building in order to compare the results obtained in laboratory conditions, with those taken in field conditions.
\end{abstract}

\section{INTRODUCTION}

As with any measurement technique, TLS measurements are not perfect and subject to errors caused by different factors affecting the measurement process. Determining the sources of errors in terrestrial laser scanning measurements is complicated due to the large number of such factors, and the fact that they are usually interlinked. the complexity of this issue is enhanced by the fact that different laser scanners use different measurement methods, for example, they operate at different wavelengths and using different beam deflection units. Careful examination of all of these factors and the errors being introduced into the data, provides a solid basis for assessing the quality of these data and the performance parameters of the scanner, which is necessary when deciding on the suitability of TLS for a particular application. The most definitive indication of the quality of the data is described by the accuracy, i.e. the degree of consistency between the measurement and the true value of the measured quantity (Iavarone 2002 r.). In TLS you can use the term "precision of the 3D coordinates of points in the point cloud" as an appropriate indicator of data quality. Because TLS is a technique for measuring area/a surface, and the final product of the laser scanning is often a 3D model, we can use the term "accuracy of the model" as an appropriate indicator of the quality of the information obtained through laser scanning. In both cases, the accuracy reflects the level of compliance between the data or the model and the real world. Sources of measurement errors include instrumental errors resulting from the construction of the scanning device. The accuracy is also affected by errors originating from both the laws of physics that affect the operation of the laser and the system directing the beam, as well as errors related to the mechanics of the scanner, i.e. the laser's optical system errors, system errors associated with sending a laser beam. Measurement errors can also result from improper data acquisition techniques, eg. an inadequate selection of measurement stations and their number, or the wrong choice of scanning density relative to the accuracy requirements. Undoubtedly, parameters such as the intensity of the reflection beam, angle of incidence and surface properties (material, surface roughness) influence the final result of the process of obtaining the point cloud and its subsequent processing (Reshetyuk, 2006). The following describes these sources of error in more detail.

\section{ANALYSIS OF FACTORS AFFECTING THE ACCURACY OF TLS}

\subsection{Review of factors affecting the accuracy of TSL}

Terrestrial laser scanning is a technique of recording data using reflectorless distance measurement. Therefore, one of the main sources of error is the reflectivity of the scanned surface - SNR (Signal-to-noise ratio). It is defined as the ratio of the emitted and reflected signals. The SNR is also affected by: the material properties associated with the electrical and magnetic permeability, the color of the surface, the laser wavelength $\lambda$, the surface roughness, which depends on the wavelength and frequency, polarization, the surface temperature and surface moisture (Thiel et al., 2004 ).

Additionally, the intensity of the reflection and the accuracy of coordinate measurements from the point cloud are directly affected by the scanning geometry. The number of elements that describe the point cloud is dependent on the density of the cloud, which in turn depends on the parameters of the scan, i.e. the angular resolution, but also on the angle of incidence and distance to the subject. The density of the point 
cloud decreases with an increasing distance and an increasing angle of incidence (Lindenbergh et al., 2005).

The angle of incidence of the beam is defined as the angle between the normal, directed toward the test surface, and the direction of the laser beam (Soudarissanane et al, 2007). The angle of incidence on the surface affects the scanning beam diameter. The ideal scanning conditions are those in which the scanner beam falls at a right angle to the scanned object. Measurement errors are also affected by environmental factors such as temperature, pressure, humidity, lighting or vibrations. The basic phenomenon that occurs in the atmosphere is the propagation of the laser beam. As a result, a deformation of the returning pulse occurs weakening of the intensity of the laser light. This is due to the occurrence of Rayleigh and Mie scattering, as well as the absorption of water vapor and CO2, O3 (Rueger, 1990). When measuring in unfavorable weather conditions, 'dropout pixel' or 'false return' phenomena can be observed. (Grantham et al, 1997)

\section{RESEARCH}

Research work was performed to analyze the impact of external factors and scan parameters to the resultant accuracy of 3D models. The work was carried out on a test field and on a real structure. Measurements were taken using the FARO Focus 3D phase scanner.

The test field consists of a selection of the most popular types of materials used in construction. These include: different types of elevation plaster, brick, sheet metal, plastic, clinker brick, glazed tile and wood (fig. 1). The samples varied in size, shape and colour. Some elements were flat, some spherical and some cylindrical. Chosen samples are shown in figure 1:

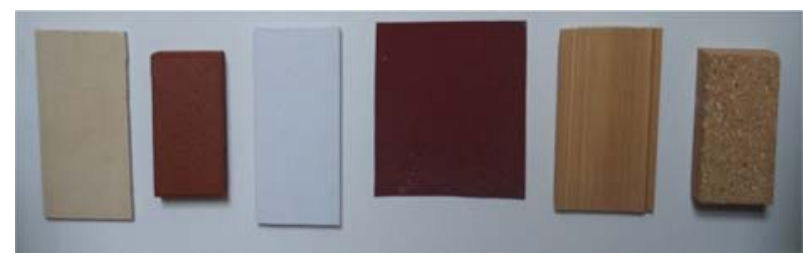

Figure 1. Test field consisting of different materials

Textures of materials were also analyzed. Examples of different types of plaster are shown in figures 2 and 3 :

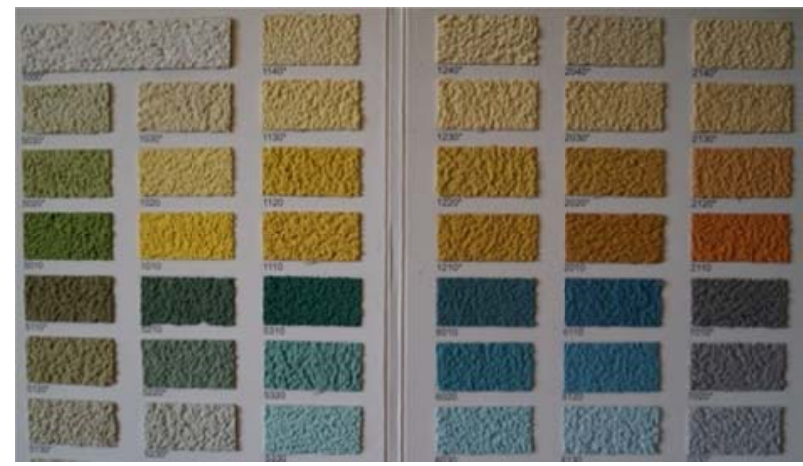

Figure 2. Acrylic plaster test

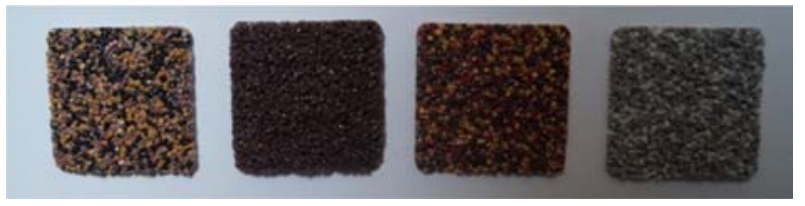

Figure 3. Grain plaster test

Measurements were taken at different incident angles of the laser's beam and from a range of distances. At first, measurements of the test field were taken at different scanning distances. The chosen distances are 5, 10, 15, 20 and 25 meters. Next, measurements were taken with different scanning angles. In this case, the scanner was set up at 10 meters from the test field and its position remained unchanged throughout this part of the experiment. The test field was then rotated by $15^{\circ}$ relative to the previous measurement. As a result the test field was measured at six different angles: $0^{\circ}, 15^{\circ}, 30^{\circ}, 45^{\circ}, 60^{\circ}, 75^{\circ}$. The scanning process was conducted with different scanning intervals: $1,53 \mathrm{~mm} ; 3,07 \mathrm{~mm}, 6,14 \mathrm{~mm} ; 7,67 \mathrm{~mm}$, in order to analyze the affect of the scanning density, the errors in fitting with the point cloud and the intensity values for varied scanning angles.

As a result, 30 scanned scenes were generated. Their initial processing consisted of filtering, the point cloud, which means eliminating redundant measurement points and noise registered by the scanner. It was crucial to remove all points which were not on the surface of the test field in order to reduce coarse errors when modelling this surface. An analysis of the modelled surfaces showed, that different samples have different errors when fitted with the point cloud, depending on the type of material making up that surface.

In order to compare the results obtained in laboratory measurements with their future practical implementation in field conditions, measurements were taken of a cultural heritage structure constructed with a number of different materials, including: light, dark and grain plaster, plastic, tiles and plastic and metal railings. All of these elements are also present on the test field and therefore it was possible to conduct a comparative analysis of the results obtained from both measurement phases.

\subsection{Analysis of the results}

A comparison and accuracy assessment of acquiring data for architectural structures was carried out on a generated 3D model for all test samples. (fig. 4)

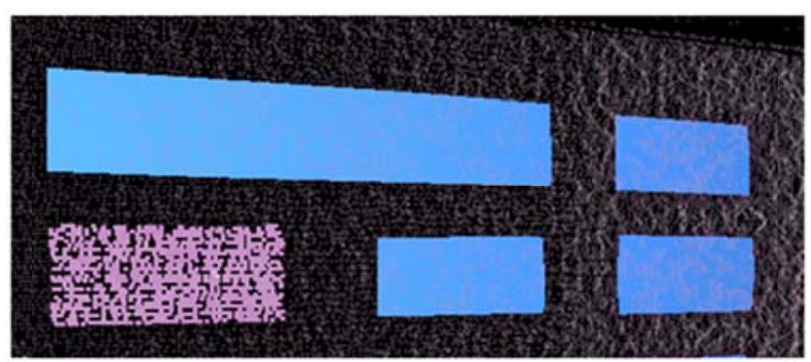

Figure 4. Model of the surface of the acrylic plaster test 
The mean value of the error in fitting the model to the point cloud was calculated, as well as the mean value of the reflection intensity. These studies have proven that the scanning distance has a very significant influence on the fitting errors.

Analyses were based on two basic scanning parameters: the angle of the beam and the distance. Graphs below show the results of these analyses.

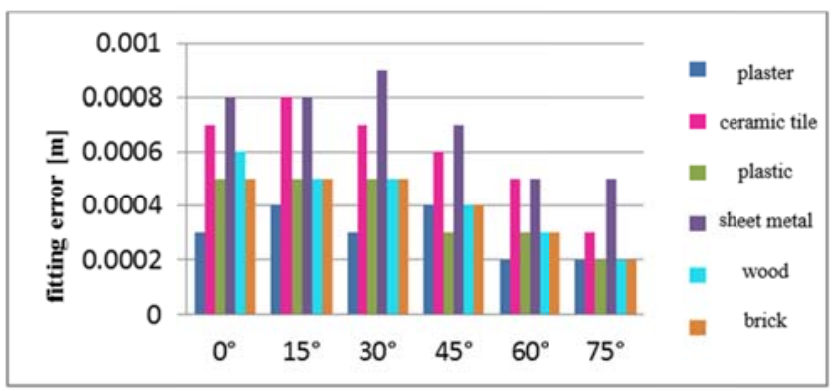

Figure 5. A plot of the fitting error of different materials in a point cloud at different angles of incidence of the laser beam on the test field

The value of the fitting error into a surface was the lowest for the ceramic plate. At low angles of incidence of the laser beam, the fitting error was in the range from $0.3 \mathrm{~mm}$ to $0,04 \mathrm{~mm}$. For angles equal to $60^{\circ}$ and $75^{\circ}$ the RMS error value reaches $\pm 0.2 \mathrm{~mm}$. Similar error values are noticeable for plastic and brick. For an angle of $0^{\circ}$ the error value is RMS $= \pm 0.05 \mathrm{~mm}$, while for $75^{\circ} \mathrm{RMS}= \pm 0.2 \mathrm{~mm}$. The materials least accurately fitted into a plane were sheet metal and clinker brick. The error obtained when the laser beam incidence was perpendicular to the test field, is in the range of from $0.7 \mathrm{~mm}$ to $0.8 \mathrm{~mm}$. When you increase the angle, errors are reduced to $\pm 0.4 \mathrm{~mm}$.

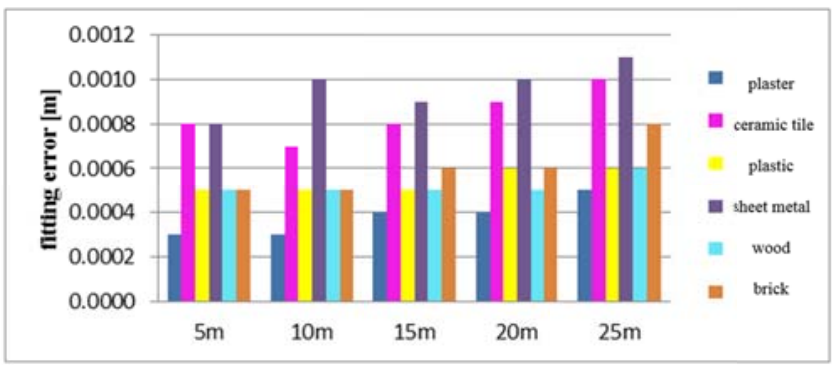

Figure 6. A plot of the fitting error of different materials in a scanned point cloud at variable distances

The chart shows that the average value of the fitting error in the point cloud gradually increases with distance between the scanner and the test field. A large randomness of results can be observed for the metal sheet, with an RMS error value at a distance of $15 \mathrm{~m}$ equal to $\pm 1 \mathrm{~mm}$, which then decreases, and then again, at a distance of $20 \mathrm{~m}$, increases to a value of \pm 1.1 $\mathrm{mm}$. Clinker brick behaves similarly, because the fitting error at $10 \mathrm{~m}$ decreases in relation to the error at $5 \mathrm{~m}$. Other materials, for example wood, are characterized by constant fitting error values for four consecutive scanning distances.

\section{CONCLUSIONS}

Studies have shown that scanning distance has a substantial impact on the value of the errors of fitting the model with measuring points. Together with an increase in the scanning distance, the number of points representing the surface of a given material decreases. The precision with which individual points are measured also decreases, as a result of which one is thicker. This 'thickness of the point cloud, determines the accuracy of the approximation of the surface of the given sample. The greater this is, the greater the fitting errors. Therefore, for the greatest scanning distance $-25 \mathrm{~m}$, the fitting errors indicate the lowest accuracy for individual samples of the test field. The conducted research shows the nature of the relationship between the reflection intensity and the distance between the scanner and the measured structure. For all of the studies samples, an increase in the distance causes a decrease in the reflection intensity.

\section{ACKNOWLEDGEMENTS}

This paper has been supported by the Military University of Technology, the Faculty of Civil Engineering and Geodesy, Department of Remote Sensing and Photogrammetry.

\section{REFERENCES}

Granthamn JW, Stargardt CD, Dungey C, Meidunas E. Laser Radar in Adverse Weather. In: SPIE Proceedings, Vol. 3065, 1997

Iavarone A., Laser Scanning Fundamentals, 2002

Lindenbergh, R., Pfeifer, N. and Rabbani, T., 2005. Accuracy Analysis of the Leica HDS3000 and feasibility of tunnel deformation monitoring. In: Proceedings of the ISPRS Workshop, Laser scanning 2005, Vol. XXXVI(3/W3), Enschede, The Netherlands, pp. 24-29

Reshetyuk Y., 2009. Self-calibration and direct georeferencing in terrestrial laser scanning. Licentiate thesis in Geodesy, Royal Institute of Technology, Division of Geodesy, 2009

Rueger, J. M.,. Electronic distance measurement. An Introduction. 3rd totally revised edition, Springer Verlag, Berlin - Heidelberg - New York - London - Paris - Tokyo Hong- Kong. 266 pages. 1990

Soudarissanane S., van Ree J., Bucksch A., Lindenbergh R., 2007. Error budget of terrestrial laser scanning: influence of the incidence angle on the scan quality. Proceedings 3DNordOst 2007, Berlin, Germany

Thiel, K.-H. and Wehr, A., 2004, Performance capabilities of laser scanners-An overview and measurement principle analysis. International Archives of Photogrammetry and Remote Sensing, Vol. 36-8/W2, pp. 14-18 